\title{
Giant radio galaxies and cosmic web
}

\author{
Pekka Heinämäki \\ Tuorla Observatory, Department of physics and astronomy, University of Turku \\ Väisäläntie 20, 21500 Piikkiö, Finland \\ email: pekheina@utu.fi
}

\begin{abstract}
Giant radio galaxies create the well distinguishable class of sources. These sources are characterized with edge-brightened radio lobes with highly collimated radio jets and large linear sizes which make them the largest individual structures in the Universe. They are also known to be hosted by elliptical/disturbed host galaxies and avoid clusters and high galaxy density regions. Because of GRG, large linear sizes lobes extend well beyond the interstellar media and host galaxy halo the evolution of the radio lobes may depend on interaction with this environment. Using our method to extract filamentary structure of the galaxies in our local universe we study whether radio lobe properties in some giant radio galaxies are determined on an interaction of this filament ambient.
\end{abstract}

Keywords. Cosmology: large-scale structure of the Universe; galaxies: structure; radio continuum: galaxies

\section{Introduction}

Among FR II radio galaxies, the largest sources of their projected linear sizes $>0.7$ $\mathrm{Mpc}$ are called giant radio galaxies (GRG). Because of their large size it is probable that the orientations of these sources are near the plane of the sky and radio lobes extend well beyond the interstellar media and host galaxy halo. As a result, we may expect that plasma of the lobes interacts with intergalactic medium (IGM) modifying morphology and their asymmetry in the lobe lengths. While GRG avoid high density regions they are assumed to be associated with large scale filamentary structures (Subrahmanyan et al. 2008) and the evolution of the radio lobes may depend on interaction with ambient gas (Safouris et al. 2009).

Relatively little is known about gas associated with galaxy filaments, but according to simulations (Cen\&Ostriker 2006) the majority of the missing baryons are hidden filaments in low-density plasma (WHIM). Low density of WHIM gas renders it very difficult to observe. While GRG location and orientation relative to filamentary structure can modify their morphology and radio properties of the radio lobes it allows the use of GRGs as intergalactic barometers (Malarecki et al. 2013).

\section{Data and Methods}

Koziel-Wierzbowska \& Stasinska (2011) analyzed FR II radio sources (the Cambridge Catalogues of Radio sources: 3C-9C) and their counterparts in SDSS DR7, finding in total 401 FR II sources. Among these sources we found 22 GRGs. As a first step in this project we study where these GRGs are located in the filament-void network. For that purpose we use Tempel et al. (2014) filament catalogue based on Bisous model (Stoica et al. 2005). This catalogue is extracted from the SDSS-DR8 and it contains 3D mapping of 15421 filaments including their properties (e.g. galaxy richness, luminosity, length). The radius of the filaments in this catalogue is fixed at $0.5 \mathrm{Mpc} / \mathrm{h}$. 

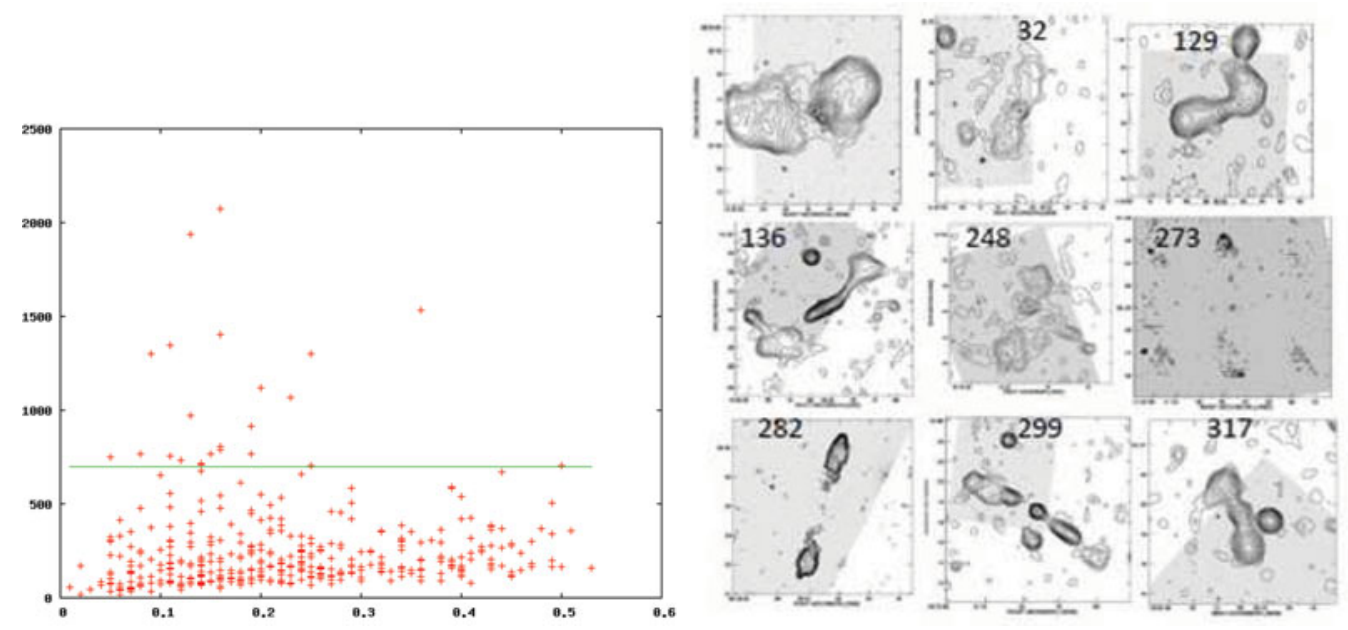

Figure 1. Linear sizes of the FRII sources in the SDSS survey region as a function of redshift. Green line indicates GRG size limit (left panel). Nine radiomaps of the GRGs that have redshifts less than $\mathrm{z}=0.155$ (right panel). Data and figure by Koziel-Wierzbowska \& Stasinska(2011)

Filament data extends up to $\mathrm{z}=0.155$ which reduce the number of GRGs to 9 . Our preliminary analysis shows that only 1 case out of these 9 GRGs has host galaxy(obj273) inside the filament (note, despite that its morphology seems to be quite symmetric). In two cases the host galaxy is in $1 \mathrm{Mpc} / \mathrm{h}$ radius from the filament axis (obj136 and obj248). We will also analyze whether the asymmetry in these sources is related to the distribution of the galaxies in the local galaxy group and what is the orientation of the nearby filament. In other six cases host galaxies and radio lobes are too far away from the filaments to have interaction with filament ambient. Note that even obj129 seems to be in a void region, it is clearly asymmetric.

\section{Summary}

The current project is at preliminary stage and the aim of this poster is to describe the idea and the starting point for more detailed forthcoming analysis of the relations between GRG and filaments. The next step is to study how radio lobes are aligned with filaments and to investigate the distribution and physical state of the gas in these regions. Moreover, we are planning to add more GRGs to our analysis.

\section{References}

Cen, R. \& Ostriker, J. 2006, APJ, 650, 60

Fanaroff, B. L. \& Riley, J. M. 1974, MNRAS, 167, 31

Koziel-Wierzbowska, D. \& Stasinska, G. 2011, MNRAS, 415, 013

Malarecki, J. M., Staveley-Smith, L., Saripalli, L., Subrahmanyan, R., Jones, D. H., Duffy, A. R., \& Rioja, M. 2013, MNRAS, 432, 200

Safouris, V., Subrahmanyan, R., Bicknell, G. V., \& Saripalli, L. 2009, MNRAS, 393, 2

Stoica, R. S, Gregori, P., \& Mateu, J. 2005, Stoch.Process.Appl., 115, 1860

Subrahmanyan, R., Saripalli, L.,Safouriys, V., \& Hunstead, R. 2008, ApJ, 677, 63

Tempel, E., Stoica, R. S., Martinez, V. J., Liivamägi, L. J., Castellan, G., \& Saar, E. 2014, MNRAS, 438, 3465 